\title{
Query Space Exploration Model Using Genetic Algorithm
}

\author{
Jae-Hoon Lee* ${ }^{*}$ Sung-Joo Lee ${ }^{* *}$ \\ * Dept. of Computer Science, Graduate School, Chosun Univ. \\ ** Dept. of Computer Engineering, Chosun Univ.
}

\begin{abstract}
Information retrieval must be able to search the most suitable document that user need from document set. If foretell document adaptedness by similarity degree about QL(Query Language) of document, documents that search person does not require are searched. In this paper, showed that can search the most suitable document on user's request searching document of the whole space using genetic algorithm and used knowledge-base operator to solve various model's problem.
\end{abstract}

Key word : Query, IR, Gene Algorithm

\section{Introduction}

Fast growth of internet is spreading fast by different industry and effort is required so that can do suitable information retrieval. Information retrieval models such as Boolean, Probabilistic, Vector space, Adaptedness feedback model had been studied interval long.

Boolean model is based on Set Theory and Boolean Algebra and offers operator of and, or, not and so on. This model is advantage that can offer clear Formalism and express meaning that user wants exactly. But, because partial matching is impossible, overfull document is searched or there is shortcoming that can not do sequencing because only minimum's document can be searched and being in difficulty degree with query is decided by 0 or 1 .

Vector space model can give proper weight(not binary) on query or keyword of document and partial matching is possible. Therefore, can do document sequencing in similarity degree of query and document.

Probabilistic model defines in model relationship and dependency between words, weight of query, similarity degree between query and document. While probability model has advantage that can define model to do formal and decide all parameters in this model, but have shortcoming that must presume early parameter.

Lately, interest was augmented to mechanical studying method. Specially, is expecting in natural selection processing by analogical inference using Neuron and evolution algorithm[1].

Gene algorithm developed by Holland and is basing on Darwin's survival theory and nature evolution processing principle[3]. Gene algorithm can improve standardization (formulation) of query, and is offering domain of information retrieval in optimized document.

This paper showed that can search for the most suitable document gathering on user's request searching several parts of

This study was supported by research funds from Chosun University, 2003 document space using gene algorithm.

\section{Basic idea of gene algorithm}

\subsection{Individual and Population}

Individual are expressed on chromosome in natural world. It is Table 1. that compare terminologies that use by gene algorithm with natural world[3].

Table 1. compare terminologies natural world and gene algorithm

\begin{tabular}{|c|c|}
\hline Natural world & Gene algorithm \\
\hline \hline chromosome & string \\
\hline gene & feature, character \\
\hline allele & feature value \\
\hline locus & string position \\
\hline genotype & structure \\
\hline
\end{tabular}

Gene operator uses selection, crossover, mutation[3].

(1) Selection

Reproduction is process that reproduce string according to fitness of each string and This time fitness function becomes any form it that want user does maximum. This process means that probability to have more sons and grandsons in next generation fitness is high individual is high.

\section{(2) Crossover}

Reproduction process search direction fitness by the highest individual head, but do not create new individual. As sons and grandsons have entirely other characteristic form and nature with parents inheriting gene from parents generation in natural world, it is crossover that imitate this process at algorithm.

\section{(3) Mutation}

Mutation process supplies new information that do not exist in present group. This is embodied changing a bit of string by 0 to 1 or by 1 to 0 accidentally to very low mutation rate 
$\left(P_{m}\right)$. This process helps can recover important information that lost at past and escapes here in case direction of search grows by regional extreme value.

(4) Evolution

Gene algorithm is used to solve optimization problem. This need gene solution and model of fitness function. Created generation's population set follows to selection, crossover, and mutation. It is proved by Goldberg that The parallel processing of gene algorithm[6].

\subsection{Research of gene algorithm in information retrieval}

Development of schema theory studied by Holland, and studied theoretically about GA.

Gordon studied better document about adaptation point gene algorithm to do descriptions[5]. Individual of an $\mathrm{N}$ are allocated on each document and each individual are consisted of set of indexing word. Make document individual that GA is good more than thing by probabilistic model. Reproduction improved $39.74 \%$ of in 20 generation and $56.61 \%$ improved in 40 generation more than thing which is produced by co-relevant document method.

Yang $\mathrm{JJ}$ and Korfhage proposed method to change weight to do document word indexing in GA and optimize query[10]. Selection used probabilistic operation method, and crossover used blind crossover that use duplex crossover point. Mutation used traditional mutation method to reproduce query population. Suitable document query is centered after 6 generation in an experiment.

Chen and Kim proposed GANNET(hybrid gene and neural network)[2]. This system is form that optimize document that user selects in keyword uses GA.

Kraft proposed GA programming to improve weight Boolean query standardization[3]. Weight Boolean query expressed on chromosome in Koza's gene model. GA's goal modifies query to improve recall factor of word and correctness rate search performance.

\section{Query Space Exploration Model Using Genetic Algorithm}

\subsection{Individual and Population}

At gene algorithm, Individual mean query and gene or chromosome is coincided index word or concept. Early locus is word weight, and early population is consisted of early question and searched suitable document list. Query of each individual appears as following

$$
Q_{u}\left(q_{u 1}, q_{u 2}, \ldots, q_{u t}\right)
$$

Query weight(qui) is calculated by query word weight scheme. Scheme is subset of all possible strings that have same bit value in decided string position. Query weight evolves through generation. Query weight formula is as following.

$$
q_{u i}=\frac{\left(1+\log \left(t f_{u i}\right)\right) \times \log \left(\frac{N}{n_{i}}\right)}{\sqrt{\sum_{k-1}^{T}\left(\left(1+\log \left(t f_{u k}\right)\right) \times\left(\log \left(\frac{N}{n_{k}}\right)\right)^{2}\right.}}
$$

$T$ displays whole move of word drawn automatically from document, and $N$ display whole move of document. $t f_{u i}$ expresses ti's frequency in document $u$. Early population $P o p(0)$ is consisted of early query and suitable document search list by early query. Find suitable document from query work to do first time for gene algorithm execution, and early population is not Random structure. Gene algorithm makes inquiries by good perfume from document space. Population is created newly after it repeats gene algorithm.

\subsection{Fitness function}

Fitness is calculated according to adaptedness of document that is given in each query of population and is searched. Fitness is proportional in near degree on documents that function is suitable and is inverse proportion in near degree on amiss documents. Fitness function formula is as following.

$$
\begin{aligned}
& \operatorname{QFitness}\left(Q_{u}^{(s)}\right)= \\
& \frac{\frac{1}{\left\|D_{r}\right\|} * \sum_{d, \in D_{r}} \operatorname{Sim}\left(d_{j,} Q_{u}^{(s)}\right)}{\frac{1}{\left\|D_{n r}\right\|} * \sum_{d_{j} \in D_{n}} * \operatorname{Sim}\left(d_{j,} Q_{u}^{(s)}\right)}
\end{aligned}
$$

$D_{r}$ means gathering of suitable document search in all generation, and $D_{n r}$ means set of document search bandit conjunction in all generation.

According to Jaccard function, Similarity value $\left(\operatorname{Sim}\left(d, Q_{u}^{(s)}\right)\right)$ is as following[3].

$$
\begin{aligned}
& \operatorname{Sim}\left(d_{j}, Q_{u}^{(s)}\right)= \\
& \frac{\sum_{i=1}^{T} q_{u i}^{(s)} \cdot d_{j i}}{\sum{ }_{i=1}^{T} q_{u i}{ }^{2}+\sum_{i=1}^{T} d_{u i}^{2}-\sum{ }_{i=1}^{T} q_{u i}^{(s)} \cdot d_{j i}}
\end{aligned}
$$

Niche's definition searches document of resemblant inherited character from query set. Niche's formula improves doing to search different document space evenly decoration, as following.

$\operatorname{Niche}\left(Q_{u}^{(s)}\right)=$

$\left\{\left(Q_{u}^{(s)}\right)\right.$ as $E D\left(Q_{u}^{(s)}, Q_{v}^{(s)}\right) \leq$ critical value $\}$

Euclid distance, $E D\left(Q_{u}^{(s)}, Q_{\nu}^{(s)}\right)$, is as following.

$$
E D\left(Q_{u}^{(s)}, Q_{v}^{(s)}\right)=\sqrt{\sum\left(q_{u i}^{(s)}-q_{u i}^{(s)}\right)^{2}}
$$

Niche's critical value is decided by underground method. Niche's weight function way is as following.

$$
\operatorname{Fitness}\left(Q_{u}^{(s)}\right)=\frac{\operatorname{QFitness}\left(Q_{u}^{(s)}\right)}{\left\|\operatorname{Niche}\left(Q_{u}^{(s)}\right)\right\|}
$$

Query Fitness is proportional in query similarity degree of suitable document in same Niche's query number and Document bandit conjunction is inverse proportional in query 
similarity degree. New domain is examined according to average fitness connected with query. Query population, $P o p^{(s)}$, is composed as following.

$$
P_{O D}^{(s)}=\left\{\left(Q_{u}^{(s)}, \text { Fitness }\left(Q_{u}^{(s)}\right)\right) u=1 \ldots \text { Popsize }\right\}
$$

\subsection{Gene operator}

In GA, kind of operator is Selection, Crossover, Mutation. In this paper, we use two Knowledge-based Crossover Operator(Term Weight Based, Term Co-occurrence Based) and Blind Crossover Operator.

\subsubsection{Selection}

Selection selects chromosome of population to two for hybridization and use roulette wheel selection traditionally. After yield ratio with relative value of conjunction of fidelity of all chromosomes and fidelity of each chromosome in population, chromosome that have of dominant character is existed more in next generation averagely because have as selected possibility as the probability and recessive chromosome is selected.

\subsubsection{Crossover}

Crossover consists of pair of individual that is selected by crossover probability value $P_{c}$. Use two intersection points selected random to create chromosome of next generation. Select position to contact according to predetermined rules after copy two chromosomes that is selected by selector and change part of chromosome each other. This time sphere that change is localized on parameter interior of selected position. After crossover, genetic factors of parents chromosome create confidence chromosome that have new argument being composed. Crossover that do fetters in word weight uses query belonging to same Niche and that do fetters to word co-occurrence uses query from other Niche.

\subsubsection{Mutation}

Mutation can prevent that fall in part optimization by select argument of dyeing interior of the body unintentionally and act role that create new argument in chromosome because exchanging the cost to love and recover genetic factor that lose at evolution front step. This paper uses two mutation operators.

\section{(1) Mutation based word adaptedness}

Change weight of specification word by weight mean value of all words in query mutation here to achieve mutation by fixed probability to words that show on query.

$$
\text { score }\left(t_{i}\right)=\frac{\sum_{d j \in D_{i}^{(j)}} d_{j i}}{\left\|D_{r}^{(s)}\right\|}
$$

\section{(2) Blind Mutation}

This operator changes weight cost by fixed probability by random value to words that show on query.

\section{An Experiment and Result}

Estimation method measured accuracy augmenters of high position 15 document through repeat of gene algorithm in this treatise. That repeat much is to search the most suitable document and estimate document order. In an experiment, Repeat fixed by 5 times and whenever repeat creates new generation through new gene algorithm. At this process, decide adaptedness about high position 15 documents that make new document list and is decided that is suitable. The goal of gene algorithm is searching for new adaptedness document through repeat of search. Method to measure efficiency of gene algorithm is shown in Table 2.

Table 2. Method to measure efficiency of gene algorithm

1. About early query search achievement.

2. Adaptedness decisions about high position 15 document.

3. Early population creation.

3.1 Add decided documents to population $\left(P o p^{(s)}\right)$ that is suitable with early query.

3.2 Adaptedness of each individual decision.

3.3 Gene operator application.

Repeat

(1) Select two parents by wheel-selection

(2) Crossover two parents by specification probability.

(3) Mutate created descendants by specification probability.

Until reach in size of population, repeat.

4. Achieve search about each query in population.

5. Search result adding up.

6. Adaptedness decisions about high position 15 document.

7. Calculate Fitness of each query.

8. Gene algorithm achievement.

9. Repeat $4 \sim 8$.

In Table 3., gene algorithm using operator of G1(based on term weight) and G2(based on term co-occurrence) to measure parameter crossover rate $\left(P_{c}\right)$, mutation rate $\left(P_{m}\right)$, population size performance experiment.

Table 3. experiment sets $P_{c} \in\{0.1,0.25,0.5,0.7\}, \quad P_{m} \in$ $\{0.01,0.07,0.1,0.23,0.5\}$, size of population by 5 . Value in parenthesis is accumulated whole summation of suitable document search. Experimented these process 5 times repeatedly, and repeat 0 is documents that is searched by early query. $P_{c}$ and $P_{m}$ act important role in information retrieval of gene algorithm.

If see in Table 3., can know that is influenced in crossover rate more than the mutation rate. If examine accumulated whole sum, displaying 259 when is $P_{c}=0.1$, and is displaying 292 when is $P_{c}=0.7$. According to result, some point of doubt occurs. Because this is influenced by mutation operator. Mutation operator is causing very small effect at gene algorithm, and use very small probability value $(0.005)$. But, this operator is very important at algorithm. Firstly, it is to use traditional gene algorithm, and second is to recombine genetic factors efficiently after crossover operation. Because this can recover damage of important genetic factor through 
crossover operation effectively. Used $P_{c}=0.7, P_{m}=0.07$ in this paper.

Table 3. An experiment by crossover rate $\left(P_{c}\right)$, mutation rate $\left(P_{m}\right)$.

\begin{tabular}{|c|c|c|c|c|c|}
\hline Repeat & 1 & 2 & 3 & 4 & 5 \\
\hline$P_{m}$ & \multicolumn{5}{|c|}{$P_{c}=0.1$} \\
\hline 0.01 & $91(91)$ & $36(127)$ & $43(170)$ & $53(223)$ & $36(259)$ \\
\hline 0.07 & $91(91)$ & $36(127)$ & $43(170)$ & $53(223)$ & $37(260)$ \\
\hline 0.1 & $91(91)$ & $36(127)$ & $43(170)$ & $53(223)$ & $37(260)$ \\
\hline 0.25 & $91(91)$ & $36(127)$ & $44(171)$ & $53(224)$ & $35(259)$ \\
\hline 0.5 & $1(91)$ & $36(128)$ & $47(175)$ & $49(224)$ & $36(260)$ \\
\hline$P_{m}$ & \multicolumn{5}{|c|}{$P_{c}=0.25$} \\
\hline 0.01 & $89(89)$ & $60(149)$ & $43(192)$ & $36(228)$ & $41(269)$ \\
\hline 0.07 & $89(89)$ & $60(149)$ & $43(192)$ & $36(228)$ & $40(268)$ \\
\hline 0.1 & $89(89)$ & $60(149)$ & $43(192)$ & $36(228)$ & $40(268)$ \\
\hline 0.25 & $89(89)$ & $60(149)$ & $43(192)$ & $36(228)$ & $40(268)$ \\
\hline 0.5 & $89(89)$ & $60(149)$ & $43(192)$ & $36(230)$ & $38(268)$ \\
\hline$P_{m}$ & \multicolumn{5}{|c|}{$P_{c}=0.5$} \\
\hline 0.01 & $92(92)$ & $49(141)$ & $49(190)$ & $48(238)$ & $37(275)$ \\
\hline 0.07 & $92(92)$ & $49(141)$ & $49(190)$ & $48(238)$ & $36(274)$ \\
\hline 0.1 & $92(92)$ & $49(141)$ & $49(190)$ & $48(238)$ & $36(274)$ \\
\hline 0.25 & $92(92)$ & $49(141)$ & $49(190)$ & $48(238)$ & $37(275)$ \\
\hline 0.5 & $92(92)$ & $49(141)$ & $47(188)$ & $46(234)$ & $34(266)$ \\
\hline$P_{m}$ & \multicolumn{5}{|c|}{$P_{c}=0.7$} \\
\hline 0.01 & $94(94)$ & $54(148)$ & $69(217)$ & $39(256)$ & $36(292)$ \\
\hline 0.07 & $94(94)$ & $54(148)$ & $69(217)$ & $39(256)$ & $36(292)$ \\
\hline 0.1 & $94(94)$ & $54(148)$ & $69(217)$ & $39(256)$ & $36(292)$ \\
\hline 0.25 & $94(94)$ & $54(148)$ & $69(217)$ & $39(256)$ & $34(290)$ \\
\hline 0.5 & $94(94)$ & $54(148)$ & $68(216)$ & $40(256)$ & $34(290)$ \\
\hline
\end{tabular}

Population size (Popsize) decides factor. Size of big population generates noise, but can execute many queries. Small size breeds silence because genetic factor transformation of reason occurs from same document space.

In Table 4., show experiment result and that unuse that use gene algorithm by size of population in operator use of G1(based on term weight) and G2(based on term co-occurrence). Lists of Table 3. are expressing value of suitable document search, and show document searched whole summation that value in parenthesis is suitable.

Table 5. is comparing each other using knowledge-based operator method and Blind operator method after fix size of population by 6 . Knowledge-based operator method is expressing efficiency of double almost more than Blind operator method.

Table 6 is expressing value of suitable document search in repeat of gene algorithm. Critical Value acts very important role in Niche operator. The best critical value appeared through $0.1 \sim 0.2$.
Table 4. Comparison of $\mathrm{G} 1$ and $\mathrm{G} 2$ by size of population

\begin{tabular}{|c|c|c|c|c|c|}
\hline & \multicolumn{5}{|c|}{ Gl } \\
\hline & iter 1 & iter2 & iter3 & iter4 & iter 5 \\
\hline \multicolumn{6}{|c|}{ Popsize $=2$} \\
\hline N GA & $90(90)$ & $48(138)$ & $33(171)$ & $46(217)$ & $24(241)$ \\
\hline$\overline{Y G A}$ & $72(72)$ & $52(124)$ & $36(160)$ & $46(206)$ & $24(230)$ \\
\hline \multicolumn{6}{|c|}{ Popsize $=4$} \\
\hline N GA & $90(90)$ & $57(147)$ & $50(197)$ & $42(239)$ & $42(281)$ \\
\hline Y GA & $96(96)$ & $64(160)$ & $40(200)$ & $45(245)$ & $42(287)$ \\
\hline \multicolumn{6}{|c|}{ Popsize $=6$} \\
\hline$N G A$ & $90(90)$ & $57(147)$ & $39(186)$ & $44(230)$ & $37(267)$ \\
\hline Y GA & $96(96)$ & $64(160)$ & $55(215)$ & $51(266)$ & $30(296)$ \\
\hline \multicolumn{6}{|c|}{ Popsize $=8$} \\
\hline N GA & $90(90)$ & $64(154)$ & $42(196)$ & $37(233)$ & $42(275)$ \\
\hline $\mathrm{Y} \mathrm{GA}$ & $96(96)$ & $72(168)$ & $50(218)$ & $38(256)$ & $29(285)$ \\
\hline & \multicolumn{5}{|c|}{ G2 } \\
\hline & iter 1 & iter2 & iter3 & iter4 & iter5 \\
\hline \multicolumn{6}{|c|}{ Popsize $=2$} \\
\hline $\mathrm{N} \mathrm{CA}$ & $90(90)$ & $59(149)$ & $36(185)$ & $40(195)$ & $21(216)$ \\
\hline Y GA & $72(72)$ & $42(112)$ & $42(154)$ & $35(189)$ & $21(210)$ \\
\hline \multicolumn{6}{|c|}{ Popsize $=4$} \\
\hline N GA & $90(90)$ & $54(144)$ & $46(190)$ & $40(230)$ & $41(273)$ \\
\hline Y GA & $85(85)$ & $40(125)$ & $46(171)$ & $58(229)$ & $45(273)$ \\
\hline \multicolumn{6}{|c|}{ Popsize $=6$} \\
\hline NGA & $90(90)$ & $64(154)$ & $40(194)$ & $31(225)$ & $31(256)$ \\
\hline Y GA & $85(85)$ & $64(149)$ & $41(190)$ & $51(241)$ & $37(278)$ \\
\hline \multicolumn{6}{|c|}{ Popsize $=8$} \\
\hline N GA & $90(90)$ & $64(154)$ & $36(190)$ & $40(230)$ & $30(260)$ \\
\hline Y GA & $85(85)$ & $64(159)$ & $45(204)$ & $36(240)$ & $30(270)$ \\
\hline
\end{tabular}

Table 5. Comparison of Knowledge-based operator and Blind operator

\begin{tabular}{|c|c|c|c|c|c|}
\hline operator & iter1 & iter2 & iter3 & iter4 & iter5 \\
\hline \multicolumn{7}{|c|}{ Knowledge-based } \\
\hline G1 & $96(96)$ & $64(160)$ & $55(215)$ & $51(266)$ & $30(296)$ \\
\hline G2 & $85(85)$ & $64(149)$ & $41(190)$ & $51(241)$ & $37(278)$ \\
\hline \multicolumn{7}{|c|}{ Blind } \\
\hline G1 & $90(90)$ & $23(113)$ & $23(136)$ & $32(168)$ & $22(190)$ \\
\hline G2 & $27(27)$ & $28(55)$ & $41(96)$ & $28(126)$ & $24(150)$ \\
\hline
\end{tabular}

Table 6. Niche result by critical value

\begin{tabular}{|c|c|c|c|c|c|c|}
\hline $\begin{array}{c}\text { Critical } \\
\text { value }\end{array}$ & iter1 & iter2 & iter3 & iter4 & iter5 & Total \\
\hline 0 & 85 & 64 & 41 & 51 & 37 & 278 \\
\hline 0.01 & 105 & 56 & 51 & 51 & 41 & 304 \\
\hline 0.25 & 94 & 60 & 51 & 50 & 37 & 300 \\
\hline 0.5 & 94 & 60 & 48 & 50 & 42 & 294 \\
\hline 1 & 88 & 64 & 54 & 42 & 48 & 296 \\
\hline 2 & 87 & 69 & 63 & 34 & 29 & 282 \\
\hline 5 & 86 & 75 & 60 & 30 & 31 & 282 \\
\hline 8 & 85 & 84 & 42 & 29 & 34 & 274 \\
\hline 10 & 85 & 64 & 61 & 47 & 25 & 283 \\
\hline
\end{tabular}


If compare G1, G2, G3, in Table 7, Niche's performance was estimated as is good. G3 is more effective in case component is discrete more than G1, G2 method. If use Niche technology in gene algorithm search, performance improved.

Table 7. Compare G1, G2, G3

\begin{tabular}{|c|c|c|c|c|c|}
\hline & iter1 & iter2 & iter3 & iter4 & iter5 \\
\hline G1 & $96(96)$ & $64(160)$ & $55(215)$ & $51(266)$ & $32(298)$ \\
\hline G2 & $85(85)$ & $64(149)$ & $41(190)$ & $51(241)$ & $37(278)$ \\
\hline G3 & $105(105)$ & $56(161)$ & $51(212)$ & $51(263)$ & $41(304)$ \\
\hline
\end{tabular}

\section{Conclusion}

In experiment result, certified that information retrieval that improve more if use gene algorithm in information retrieval system.

This paper implements and experimented following things. First, create query population newly. Second, it is able to search different sacred ground by probability from document space. Third, it is using Knowledge based operator not Blind operator that use by general gene algorithm. Fourth, it is used Niche technology to search documents that is discrete to different document space.

Experiment result depends on probability values of much parameter, size of population, critical value and produces result through gene algorithm. This paper shows in information retrieval how gene algorithm can be utilized. Gene algorithm is many problems in external form. Direct comparison did not with other Relevance Feedback method. But, through an experiment, GA can be used usefully to IR. Specially, capacitate estimate which may show good performance from document set that documents of mixing(heterogeneous) collect.

\section{Reference}

[1] Boughanem $M$ and Soule-Dupuy, Query modification based on relevance backpropagation. In: Proceedings of the 5th International Conference on Computer Assisted Information Searching on Internet, Montreal, pp. 469-487, 1997.

[2] Chen Machine learning for information retrieval: Neural networks, symbolic learning and genetic algorithms. JASIS, 46(3):194-216, 1995.

[3] Davis Handbook of Genetic Algorithms. Van Nostram Reinhold, New York, 1991.

[4] Haines D and Croft WB Relevance feedback and inference networks. In: ACM SIGIR International Conference on Research and Development in Information Retrieval, pp.2-11, 1993.
[5] Gordon M Probabilistic and genetic algorithms for document retrieval. Communications of the ACM, pp. 1208-1218, 1988

[6] Goldberg DE Algorithmes genetiques. Exploration, optimisation et apprentissage automatique. addison Wesley, France, 1994.

[7] Harmaqn D TREC overview. In: 6th International Conference on Text Retrieval TREC6, November 21-23. Harman DK,ed. NIST SP, PP. 1-24, 1997.

[8] Robertson $S$ and Walker $S$, On relevance weights with little relevance information. ACM/SIGIR International Conference on Research and development in Information Retrieval, pp. 16-24, 1997.

[9] SEbag $M$ and Schoenauer $M$, Controle un algorithme genetique. Revued' intelligence artificielle, 2/3:389-428, 1996.

[10] Yang JJ and Korhage R, Query optimization in information retrieval using genetic algorithms. ICGA, 1993.

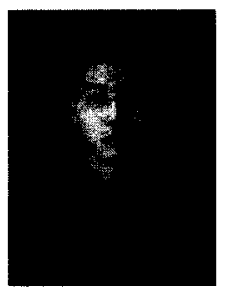

Jae-Hoon Lee

2000 : Dept. of Computer Sciences, Chosun University(B.S)

2002 : Dept. of Computer sciences, Chosun University(M.S)

2002 : Dept. of Computer Science Chosun University Doctoral Student

Research Interests : Software engineering, Programming Language, Object-oriented software, Genetic Algorithm, eCRM

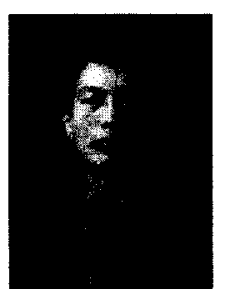

Sung-Joo Lee

1970 : Dept. of Physics Sciences, Hannam University(B.S)

1992 : Dept. of Computer sciences, Kwangwoon University(M.S)

1998 : Dept. of Computer sciences, Catholic University of Daegu(Ph.D) 1988 -1990 : Chief, Computer Center, Chosun University

1995 1997 : Dean, Information Science, Chosun University

$1981 \sim$ now : Professor in the Dept. of Computer Engineering, Chosun University

Research Interests : Software engineering, Programming Language, Object-oriented software, Rough set 